\title{
Integrated context-aware driver assistance system architecture
}

\author{
Elhadi M. Shakshuki ${ }^{\mathrm{a}, \mathrm{b}, *}$, Wael Alghamdi ${ }^{\mathrm{a}}$ and Tarek Sheltami ${ }^{\mathrm{c}}$ \\ ${ }^{a}$ Jodrey School of Computer Science, Acadia University, Wolfville, NS, Canada \\ ${ }^{\mathrm{b}}$ King Faisal University, Hofuf, Saudi Arabia \\ ${ }^{\mathrm{c} C o m p u t e r}$ Engineering Department, King Fahd University of Petroleum and Minerals, Dhahran, \\ Saudi Arabia
}

\begin{abstract}
Recently, significant improvements have been made in the area of vehicular communication systems. Furthermore, vehicle-to-vehicle communication is considered a key concept for keeping roads safe. An efficient implementation of these systems is necessary to ensure the safety of driving situations and to reduce the collision rates. This paper proposes a ContextAware Driver Assistance System that links drivers with the physical environment surrounding them using multiple types of sensors and traffic systems as well as considering the senior driver's difficulties and the system processing time. This is achieved by developing a warning system that assists drivers to avoid collisions and improve their response times. The proposed system architecture consists of a set of components to process the user's request such as parking assistance, and to provide responses and advices when needed. These components include communication, knowledge exchange, knowledge update, and contexthistory. Also, it includes other processes such as context-history manipulation, hazard detection, and hazard detection control. The main goal of the proposed system is to reduce the number of car accidents and improve driver's decisions. The NXT Robotic environment is used to demonstrate the feasibility of the proposed system.
\end{abstract}

Keywords: Context-aware system, advanced driver assistance system, vehicle-to-vehicle communication, lego mindstorms NXT

\section{Introduction}

A recent study shows that there are between 2800 to 2900 people killed on Canadian roads each year and approximately 160,000 road accidents [13]. Nowadays, there are over 1.27 million car accidents worldwide [18]. Transport Canada [29] shows that 2011 fatal collisions on Canadian roads while the total injuries was 172,883 people in the year of 2009 . To most people in society, the automobile industry provides recognizable value in regards to improvements in safety and efficiency. Accidents are considered one of the most detrimental aspects in the usage of automobiles. Ordinarily, these accidents stem from driver errors such as speeding and aggressive driving. In fact, more than $90 \%$ of the accidents happened because of driver error. The costs of these accidents in terms of human life, injuring and property are shocking [12].

One study [5] shows that in 2001, there were 42,900 people killed due to car accidents. The study showed an interesting pattern, which concluded that every 12 minutes someone died, and every 14 seconds people were injured. The study showed that most car accidents affected a specific age group,

\footnotetext{
${ }^{*}$ Corresponding author: Elhadi M. Shakshuki, Jodrey School of Computer Science, Acadia University, Wolfville, Nova Scotia, B4P 2R6 Canada. Tel.: +1 902585 1524; Fax: +1 902585 1067; E-mail: elhadi.shaksuki@acadiau.ca, eshakshuki@ kfu.edu.sa.
} 
those between the group age of 15 to 24 , as well as drivers over the age of 75 years. Drivers were not only the victims, pedestrians were also affected; the estimated number of pedestrians death was around 5,800 death and 90,000 injured. One relevant cause of accidents showed that 3 people in every 10 Americans were driving under the influence of alcohol. More surprising, about 800 pedestrians were killed by colliding with a car.

Most of these accidents happen due to drivers' behavior and poor infrastructure. We, as a society, need to find an immediate solution such as building a system to help reduce accidents by linking driver awareness to infrastructure and to the surrounding area. The proposed system should make drivers pay more attention to the surroundings and allow them to communicate with other entities of the environment such as vehicles and drivers [7]. Vehicular safety will be enhanced by the acquisition of more information about the driving environment. Consequently, the system proposed will be able to alert drivers before an accident happens; for instance, driving over the speed limit, or handling drivers' errors, or pressing the brakes late or early.

An immediate action should be taken to decrease car accidents. Based on the information provided above, most of car accidents happened due to driver's behavior and wrong decision-making. According to this, the proposed system architecture should be able to assist drivers and alert them for any upcoming hazards. Presently, Advanced Driver Assistance Systems exist and solve the problem using different architectures. This paper will identify some of the problems found in the existing systems and solve these issues using different methodologies. These problems are:

1. Multiple sensors of the same type: most of the existing ADAS systems are relying on multiple sensors from the same type. One external factor may overload the system and make it unable to function well.

2. System processing time is high: Existing systems repeat the same process for similar driving situations.

3. Ignoring traffic systems: Traffic systems provide valuable information. However, they are ignored by ADAS systems.

4. Ignoring senior drivers: senior drivers spent a significant amount of time looking to the system's interfaces. Nevertheless, seniors were not considered in previous systems.

The main idea behind this research is to propose a system architecture, which can handle a group of advanced driver assistance system functions to work together as a coherent system and solve the aforementioned problems. This system will link drivers with changes in the physical environment, improve their driving behavior, and reduce driving errors. There have been many attempts to reduce car accident rates by improving driver's behavior and their response time by developing context-aware systems. However, the proposed system architecture in this paper provides a solution from a different perspective. The main objectives of this research are as follow:

1. To investigate the major reasons that cause car accidents.

2. To study the existing work in the area of advanced driver assistance system.

3. To propose a context-aware driver assistance system architecture that combines many ADAS functions to help drivers in their decision-making and improve human behavior and response time, as well as considering the aforementioned problems.

4. To demonstrate the viability of the proposed system architecture using NXT robots environment.

\section{Background}

This section is introducing context-aware systems and its concept as well as advanced driver assistance system functions and features. Also, there is a study about standard and guidelines which illustrated the 
system concept and the interface concept. These concepts need further explanation before attempting to introduce the proposed system architecture.

\subsection{Context-aware systems}

Context-aware system is a system that can sense the physical environment [3]. The physical environment is a very important factor to consider when attempting to design equipment capable of improving the driver's decision-making or behaviour because it is exteremely dynamic environment $[9,10,27]$. Recently, significant improvements and models were developed in the area of vehicular communication systems $[2,6,8,21,25]$. In fact, vehicle-to-vehicle communication (V2V) and vehicle-to-infrastructure (V2I) are considered as a key concept to keep roads safe [11]. Implementation of these systems needs to be efficient enough to ensure safety and reduce car collision rates. From a research point of view, modeling a context-aware driver assistance system can greatly assist in the development of cars and improve drivers' behaviour/decision making processes.

\subsection{Advanced driver assistance systems}

Sensors are the major component on the application of Advanced Driver Assistance System (ADAS) while ADAS functions are the backbone of the system. A study on ADAS is presented in [4,20]. In Pijpers's study [20], the author summarizes some of the functions and sensors used in ADAS. These functions include Night Vision (NV), Departure Warning (DW), Forward Collision Warning (DCW), Curve and Speed Limit (CSL), Adaptive Cruise control (ACC), Lane Keeping Assistance (LKA), Pedestrian Detection (PD), Parking Assistance (PA), Traffic Sign Recognition (TSR), Intelligent Speed Adaption (ISA), and Environment Reconstruction (ER). Functions briefly explanied in the proposed system architcture section.

\subsection{Car of the future}

ADAS for the cars of the future was introduced by Thalen in [25]. Thalen discusses the interface concepts for Advanced Driver Assistant Systems in a sustainable mobility concept projected for 2020. The author attempts to provide standards and guidelines for the next generation who are interested in developing an advanced driver assistance system. System concept and interface concept are two points that Thalen introduces which need further discussion.

System concept: Every design of an Advanced Driver Assistance System should start with an investigation and analysis for the driver's needs. Many drivers will accept Advanced Driving Assistance Systems under the conditions that they still have control over their vehicle. Normally, drivers want the system to assist and guide rather than take the control out of their hands [25]. To meet the driver's expectations and requirements, researchers and designers should make sure that their systems and approaches provide a certain amount of control over the vehicle for the driver. Otherwise, the driver will not be involved in the driving experience. At this point, the typical vehicle consumer will either not purchase a car with an ADAS installed or turn off the system if they have already purchased it. The logic behind the system is to improve driver errors and avoid bad decision-making under stressed/extraneous circumstances. Presently, the functions of these systems are well known [2,9,25]. Significantly, the primary goal behind the system is to improve safety and increase the flexibility of the driving tasks.

Interface concept: The interface component plays a key role in making the task of driving comfortable and to decrease driver distraction. In order to develop an efficient system, three interactions must be taken 
into consideration. They are the interaction between the driver and the system, the interaction between the system and the environment, and the interaction between the vehicle and other vehicles [25]. All of these interactions are of equal importance. To illustrate these interactions, three different scenarios are considered: 1) In scenario 1, there is an interaction between a driver and a system would be if the driver disobeys the speed limit and he/she is informed of their actions by the system. 2) In scenario 2, there is interaction between a system and the environment, such as if the sensors detects a fallen tree in the driver's path and assisted in avoiding the collision. 3) In scenario 3, there is an interaction between the vehicle and other vehicle if the system recognizes there are other cars in the surrounding area and examines their speed, directions, and locations.

\section{Related work}

Many researchers attempt to develop hardware and software in the application of Advanced Driver Assistance System in order to reduce the number of future car accidents. Researchers attempt to reduce car accident rates from many different perspectives. They attempt to create a system, which can handle some functions of ADAS. The purpose of creating that system is to ensure accuracy and service quality. Recently, some car companies have already attempted to develop some functions of ADAS, but these functions are costly and work independently. For example, BMW developed parking assistance and other independent functions [19]. This indicates that there is no system that includes all necessary functions. The following subsections discuss recent research and attempts, which provide the basis for the development of system architecture to reduce car accidents

Coughlin et al. [6] provided an interesting framework for a vehicle safety/wellness system. Coughlin et al. stated that one of the most important factors for improving drivers' awareness was displaying information to the driver in regard to the surrounding area. Also, the provided framework offered in-vehicle systems to refresh the drivers' view. Thus, improving driver's performance and increasing the level of the safety. The proposed framework consists of three wellness-stimulated mechanisms. Its components offer the following services:

1. Detect component: constant monitoring for the driver's condition as well as monitoring the surrounding environment.

2. Display component: to provide this information to the driver.

3. Refresh component: to issue an alert whenever it becomes necessary. The alert calmly notifies the driver of any impeding danger then provides the driver with a second message that displays a refreshed picture showing the current driving situation.

In 2007, a proposed system architecture was introduced by [23]. This system consisted of knowledge broker, utility-based knowledge exchange, Reasoner, and interfaces. In Fig. 1, a communication component is introduced with a specific node. This component is utility-based knowledge exchange. It receives situational information, which can be defined as any information that can be used by the system to access a situation and measure by various sensors, to exchange information with other vehicles.

The knowledge broker is responsible for storing and saving situational information. The Reasoner is in charge of dealing with uncertainty, such as noise in the sensors data, failure of reading data or insufficient information. It contains five components. The first one is inductive learning based on a Bayesian network to update the structure. The second one is the prediction component, which is responsible to compute the probabilities distribution. The third component is consistency, which is responsible for checking incorrect data that comes from sensors. The term of consistency remains a vague component of the 


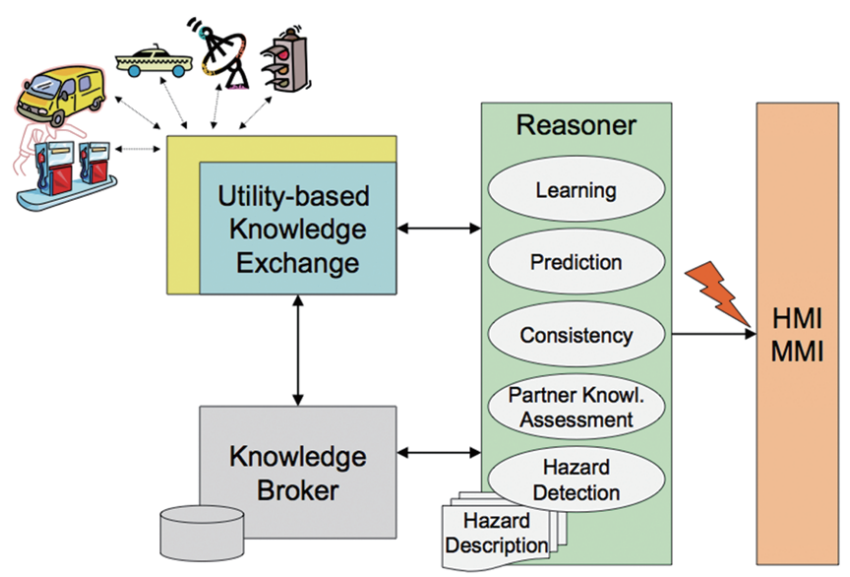

Fig. 1. Situation-aware driver assistance core components.

system, because the authors do not explain how the system would be able to check for incorrect data from its sensors. Partner utility assessment is the fourth component, which is responsible for providing information to the utility-based in order to help that component to choose a suitable network to make the communication. Finally, hazard detection is in charge of predicting any hazardous situation as soon as possible. Indeed, the suggested system architecture seems to be quite complex.

Rakotonirainy [21] believes that there is a complex interaction between the driver, vehicle, and the environment. He suggested that several types of sensors are needed in order to make the interaction valuable. One of his challenges was how to simplify the driving task to reduce the amount of errors. His approach does provide different types of information using different types of sensors within the system. He attempted to improve the driver's behavior by minimizing the number of errors [21]. Driving tasks need an immediate and appropriate decision. His proposed system focuses on the interaction between the driver, the environment, and the vehicle in order to observe and understand the driving situations using a Bayesian network. The main drawback of this system is that the Bayesian network evaluates future behavior only. We believe that the best way is to combine advanced driver assistance functions in one system to produce the best performance.

Moreover, researchers in [1] noticed that developing advanced driver assistance system employing several functions would be extremely useful for drivers to avoid car accidents. They proposed an integrated driver assistance system that utilizes image sensors [24]. In their proposed system, several functions would be employed, such as adaptive cruise control, blind spot detection, and night vision system. Using image sensors is very important to provide the driver with the required information and warns the driver whenever necessary. In [1,2], they described the most important information, which needs to be collected, such as other vehicle's speed, direction, distance, size and type. All of this information is necessary to utilize these functions. Also, having all functions working together in one coherent system is useful for drivers [1,2]. Although their proposed system could handle a great collection of functions, it is based on utilizing five cameras only. In a situation, such as a snowstorm, the system is overloaded and could not function, as it should.

\section{Context-aware driver assistance system}

The architecture of the proposed system is designed to help drivers to avoid accidents. Figure 2 shows the main components that constitute the proposed architecture, including the user interface which helps 


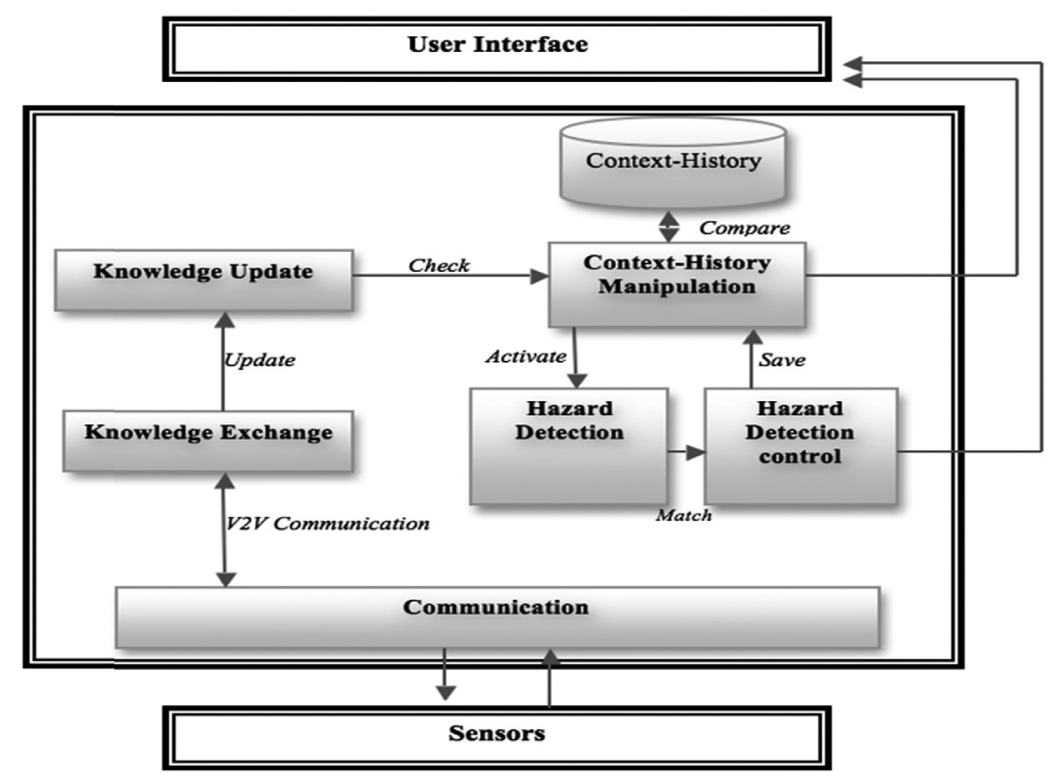

Fig. 2. Context-aware driver assistance system architecture.

the driver to enter his/her own information such as name, age and receive responses/warnings from the system. Context-History $(\mathrm{CH})$ is the database of the system that stores all previous data collected from sensors in the form of scenarios with the associated actions taken in previous scenarios.

Context-History Manipulation (CHM) component main function is to analyze and compare the current situation of the driving status with the existing scenarios previously saved in $\mathrm{CH}$. Hazard Detection (HD) component consists of a set of functions such as Adaptive Cruise Control and Lane Keeping Assistance. Hazard Detection Control (HDC) component is responsible to formulate the appropriate message and alert the driver with the current situation. Knowledge Exchange (KE) component main responsibility is handling all communicated messages between vehicles, i.e. vehicle-to-vehicle (V2V) communication; while the Knowledge Update (KU) component is responsible for updating the system's knowledge. Finally, sensors are the main sources for collecting the desired information from the surrounding area. The components of the architecture are described in more detail in the following subsections.

Figure 2 shows the proposed system architecture helping drivers to avoid accidents. The proposed system made an attempt to solve the aforementioned problems in the introduction using different concepts. They are as follow:

1. Multiple sensors from the same types: Instead of proposing a system that solely dependent on cameras or image sensors, this system used different types of sensors such as Rader, Sonar, and Laser.

2. System processing time is high: Context-History and Context-History Manipulation are expected to achieve greater processing speed. In a case of similar sensory data, the Context-History Manipulation will check the database for similar scenarios and bring the actions without performing the calculation.

3. Ignore traffic systems: Collecting data from a traffic system such as a centralized computer is introduced through the Intersection Coordination function in the Hazard Detection component. This data provide drivers' with the order deciding who goes first, second, and so on. 


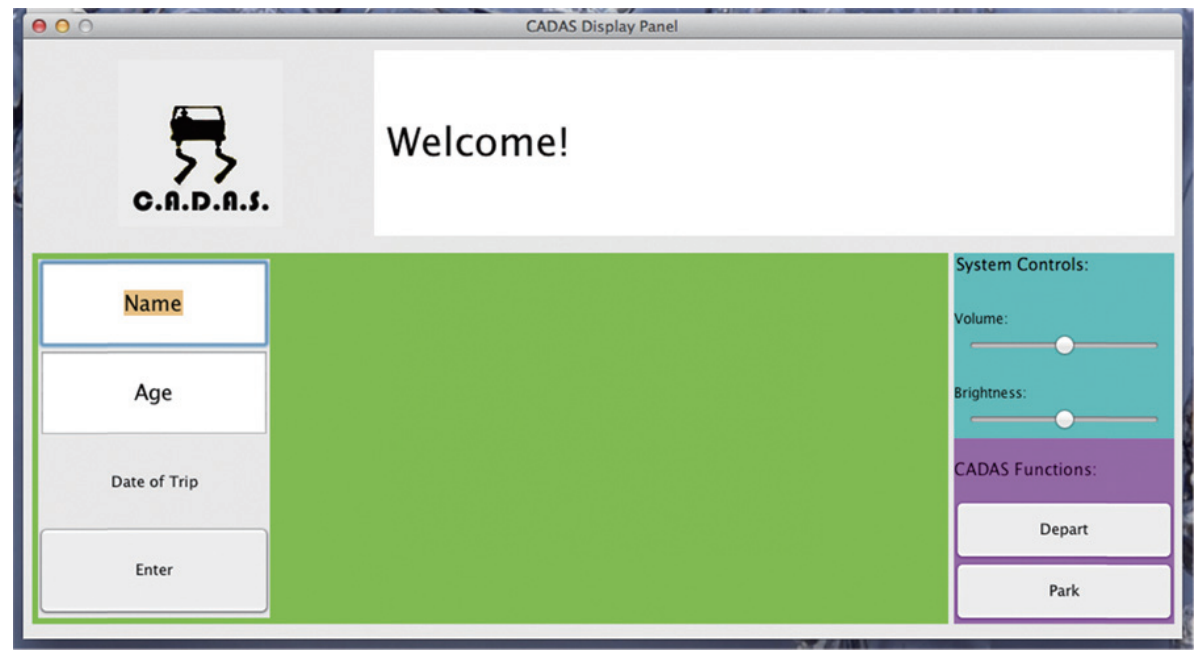

Fig. 3. Graphical user interface.

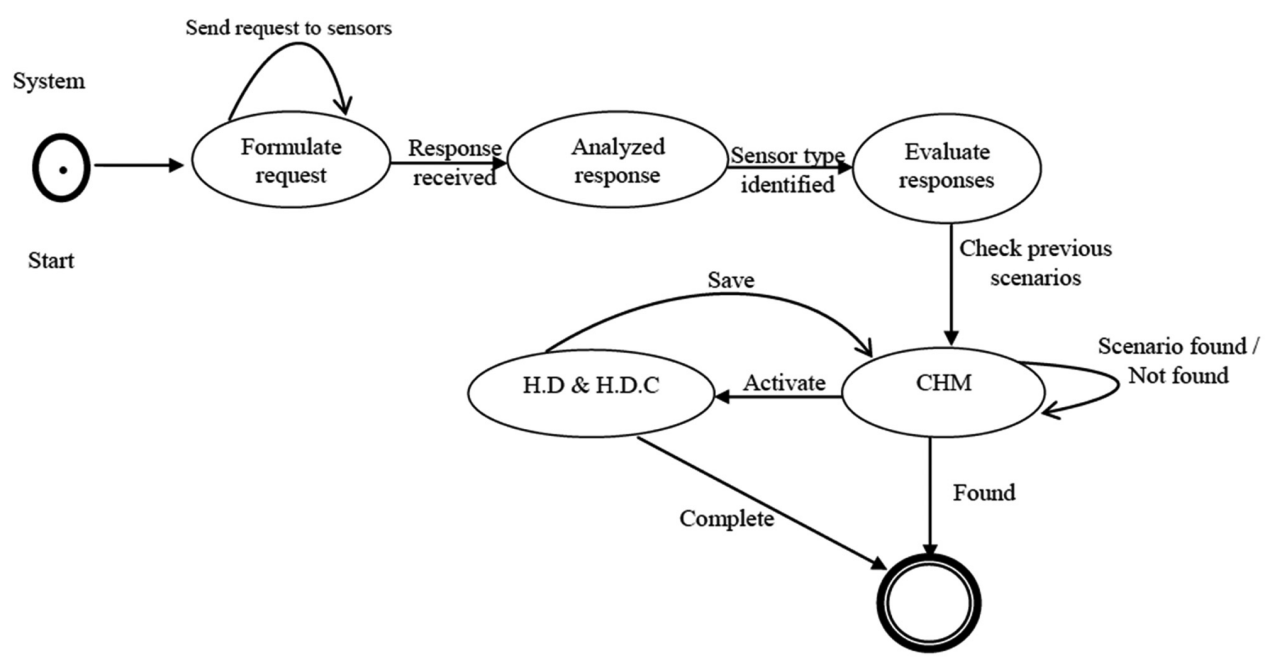

Fig. 4. Finite state representation for the system.

4. Ignore senior drivers: Hazard Detection Control is responsible to issue visual and audible suggestions through a LCD panel. This will make senior drivers understand the situation without needing to look at the LCD panel and react accordingly.

\subsection{Communication component}

The communication component of the system is responsible for sending messages to the driver, and interpreting messages received from sensors from the outside of the car to inside and vice versa. The communication can be established between this system and other systems from the same type, the system and sensors, and the system and the driver. The communication between the proposed system and the driver is established through graphical user interfaces. An example of a user interface is shown in Fig. 3. 


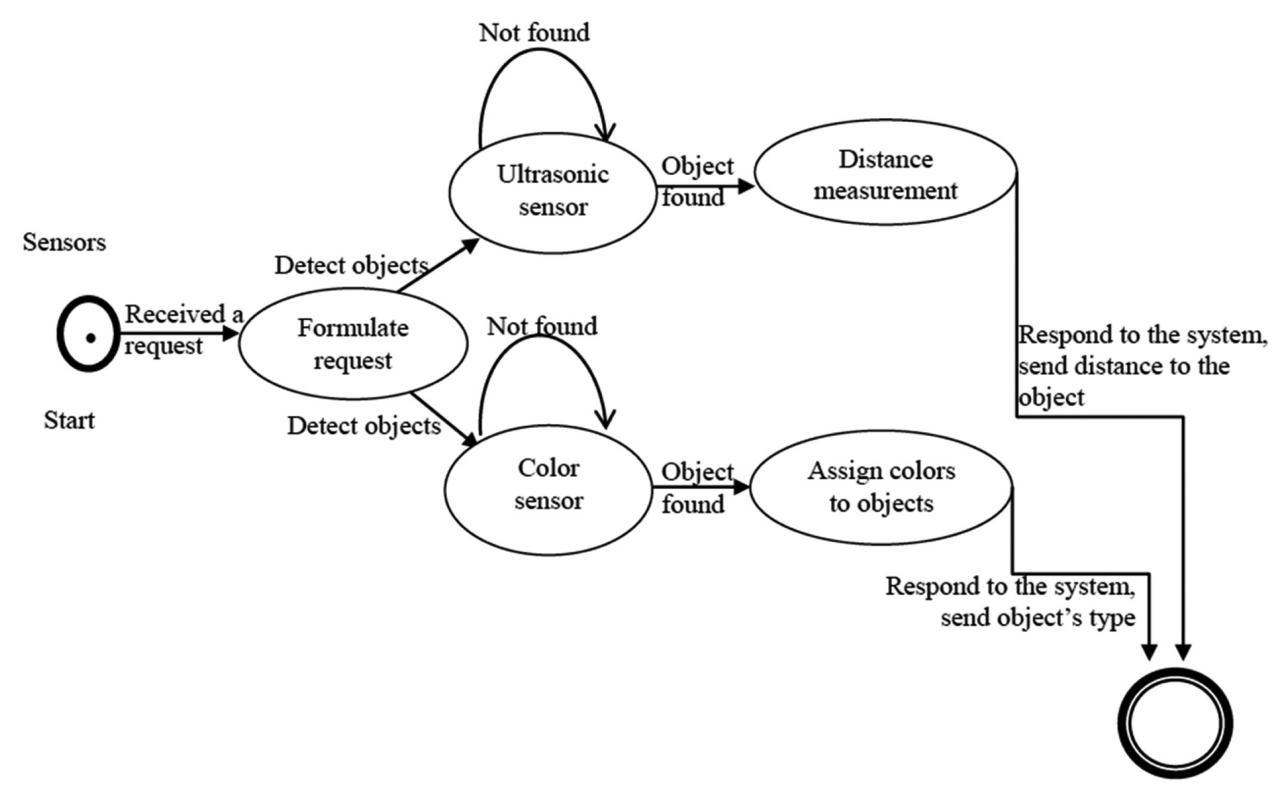

Fig. 5. Finite state representation for the sensors.

Through this interface, the driver is allowed to enter his/her name, age and date of the trip through buttons located on the left-hand side panel. There are two buttons on the right-hand side panel namely, Depart and Park. The driver can use the depart button to depart from a parking lot and use the park button to request parking assistance. The drivers are also able to adjust the volume and the brightness of the screen using the sliding buttons on the system control panel.

The communication between the system and the sensors are described by finite state representations in Figs 4 and 5. Figure 4 shows the process when the system sends a query requesting the sensors for information about the other objects, vehicles, and distances to objects in the surrounding area. The system formulates the request to sensors and sends it out in the format of $<$ sensor type, information $>$, for example, the sensor type is ultrasonic and the information is distance, so it comes as $<$ Ultrasonic sensor, distance $=d>$. When the system receives the response back, it is analyzed by CHM component to identify the sensor's type. Then, the CHM component evaluates the response utilizing the information in $\mathrm{CH}$. If data is found, then the action exists. If data not found, then activate the HD and HDC components to retrieve the appropriate actions for completing the process. An example of an action is 'Stop' or 'Reduce speed to $50 \mathrm{~km} / \mathrm{h}$ '.

Figure 5 shows how the sensors receive the system's request through the communication component. As soon as a sensor of any type receives the request, it formulates the response and sends it back to the communication component.

\subsection{Knowledge exchange and knowledge update components}

The knowledge exchange component is responsible for message exchange between vehicles within the proximity of the used sensors, which is called vehicle-to-vehicle communication [23]. Knowledge exchange provides the system with up-to-date knowledge through exchanging information and building the complete knowledge for the driver. The communication between vehicles in our experiments is established by Bluetooth connection. 


\subsection{Context-history and context-history manipulation}

One of the main components of the proposed architecture is the Context History Manipulation (CHM). The design of the functions of this component is similar to that presented in [3,23], with the main task to analyze and compare the current situation of the driving status with the existing scenarios previously saved in Context-History $(\mathrm{CH})$. If there is a similar scenario stored in $\mathrm{CH}$, then the driver is notified by sending a message that contains the action. Otherwise, the CHM passes the data to the Hazard Detection (HD) component and Hazard Detection Control (HDC) component to save the actions after it processes the scenario in this format <sensor type, information, action>. The goal of the database and CHM is to add a speeding feature in the future by saving the system processing time. If it matches previous scenarios, there is no need to activate the hazard detection and hazard detection control components. It may be useful for people who are commuting to work every day at the same time and to the same place. This implies that those systems should be faster than usual; because, there is no need to activate HD and HDC to produce an action, which eventually saves the system's processing time. The contexthistory manipulation component decides whether the current set of inputs, for example the particular sensor detects a certain object in the same distance, about the environment matches previous recorded scenarios, or not. If it did not match, it should activate the HD and HDC components to determine the course of actions to take and convey it to the driver through the graphical user interface. The course of action is determined by looking up the hazard detected in a precompiled table of responses.

\subsection{Hazard detection and hazard detection control}

Hazard Detection (HD) component is the core component of the proposed system architecture. It consists of a set of functions listed below and is responsible for detecting hazardous situations using sensors attached from inside and from outside the vehicle. The design approach of this component is similar to the approach presented in [20]; in addition to that a new function entitled Intersection Coordination is introduced. The mythology of this function is to search for vehicles in the range of an intersection using centralized computer [15]. These functions are extremely beneficial for drivers, because they improve the driver's behavior and provide the expected suggestions to different situations [25]. The following list briefly discusses the functions provided by HD component.

- Adaptive Cruise Control function: To maintain the vehicle speed and issue warnings when necessary.

- Night Vision function: To display the surrounding view during night and highlight objects on the display panel; issue warnings if the system malfunctions. It is a function that is more useful to seniors [22].

- Pedestrian Detection function: Saving human life is the main purpose behind the system. The main task for this function is to detect pedestrians and warn drivers about any hazardous situation.

- Blind Spot Detection function: To detect blind spots of both sides of the vehicle, and issue warnings if it is dangerous to change lanes.

- Parking Assistance function: To calculate all distances from the surrounding objects and issue warnings whenever there is a threat of collision.

- Traffic Sign Warning function: To read traffic signs and to ensure appropriate driver's actions, and issue warnings if the signs are disregarded.

- Forward Collision Avoidance and Speed Adaption function: To provide the distance of objects or vehicles in front of the vehicle and issue warnings, while it adapts the required speed depends on how far an object or a vehicle is from the vehicle. 


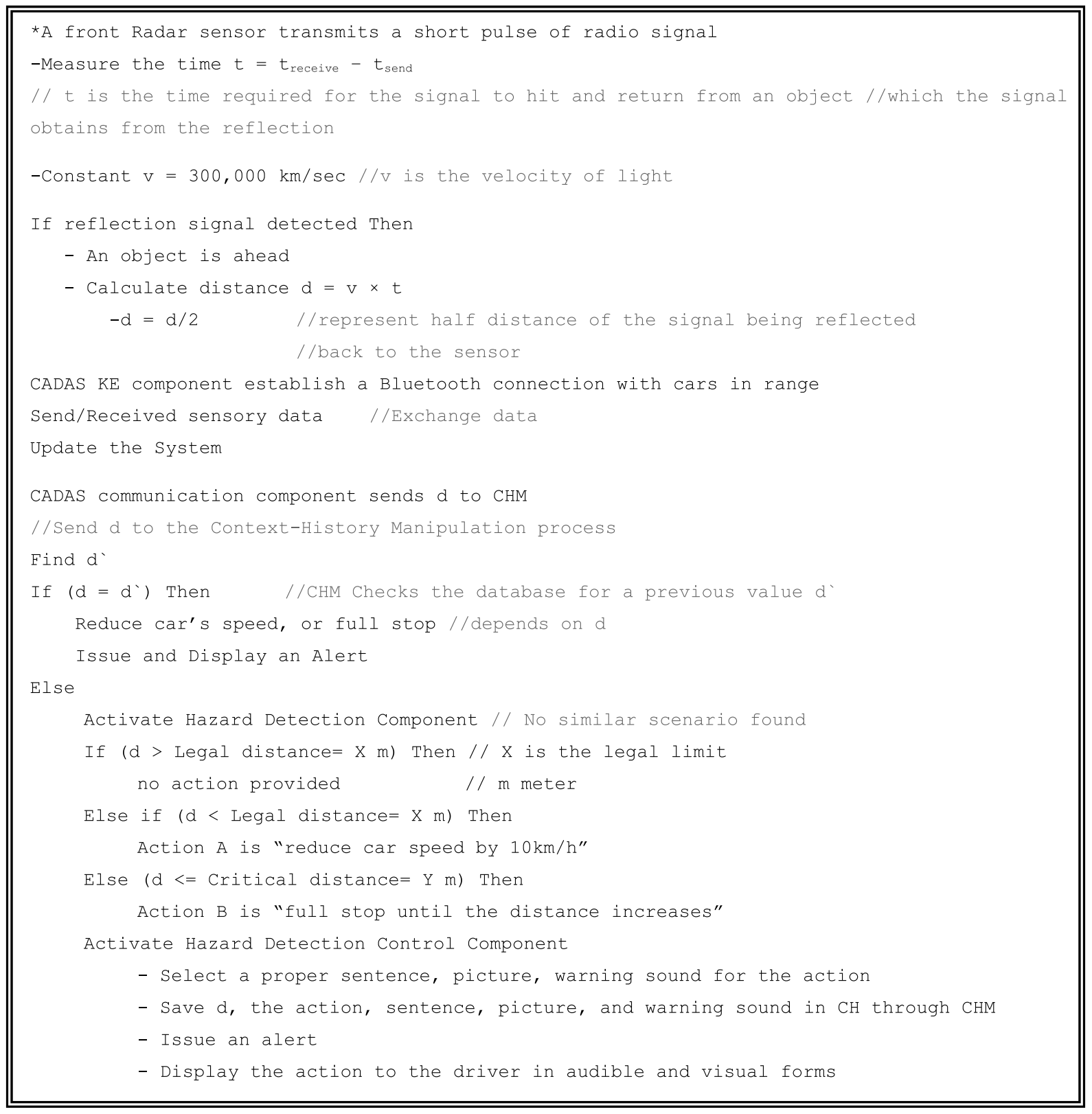

Fig. 6. Forward collision avoidance and speed adaption algorithm.

- Departure Warning function: To read marks on the roads and predict vehicle's departure path, and also issue warnings if there is an object blocking the vehicle path.

- Lane Keeping Assistance function: To help the driver stay on the right lane. Honda developed this function on 2009 [16].

- Intersection Coordination function: To establish communication with other vehicles, and provide information about intersections ahead and to decide who should go first [14]. 


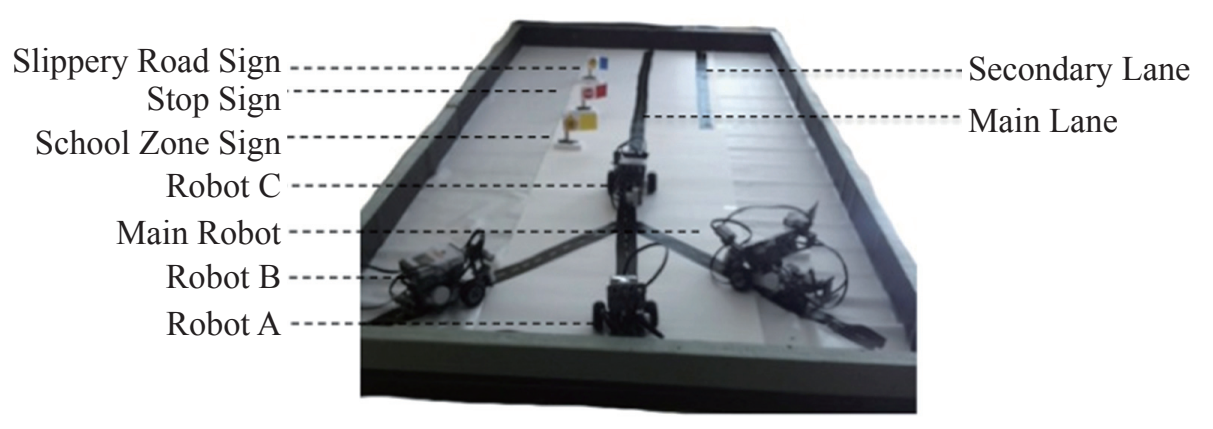

Fig. 7. The environment.

Hazard Detection Control (HDC) component is responsible for organizing the functions' results from the Hazard Detection (HD) component. If it receives a positive response from any function indicating that there is impending danger, then a warning message is issued [28]. Each result received from the HD is matched with a particular sentence and specific warning sound. The sentences are formulated in short forms and in a manner understandable to the driver. For example, if there is a car in the blind spot, the following message is displayed "avoid lane changing" or "unsafe lane changing". Indeed, this component receives the results and displays messages to the driver in an organized manner. Then, the action is represented in both audio and visual forms on the LCD panel; meanwhile, the same data is sent to the context history manipulation component for future use. Figure 6 describes an algorithm for Forward Collision Avoidance and Speed Adaption function with the process of the context-history, context-history manipulation, hazard detection, and hazard detection control.

\section{The simulation}

In an attempt to enhance and complement a greater research work, this section concentrates on the design and the simulation of a subset of the proposed functions. To demonstrate the viability of the proposed system, a simulation of a road conditions environment was designed using a Lego Mindstorms NXT Kit [26]. A few functions of CADAS are demonstrated using NXT robots [17], and designed with the capability of recognizing the status of the surrounding environment. Each function's goal was carefully examined and reaction measured to simulate changes in that environment. Figure 7 shows the environment used to simulate the functions which are Lane Keeping Assistance, Forward Collision Avoidance and Speed Adaption, Blind Spots Detection, Intersection Coordination, and Traffic Signs Recognition. The black electric tape represents the road, there were four traffic signs printed for the robots to interpret. A "stop sign", a "school zone" sign, a "slippery road" sign, and an "intersection ahead" sign were represented by the colors red, yellow, blue and green respectively. Figure 7 shows the environment.

Five functions were implemented for this simulation, Lane Keeping Assistance, Forward Collision Avoidance and Speed Adaption, Traffic Signs Recognition, Blind Spots Detection, and Intersection Coordination. A total of four Lego NXT Mindstorm robots were used in the simulation using the following configurations: Main Robot, Robot A, Robot B, and Robot C. The Main Robot was outfitted with a color sensor for traffic sign recognition, a light sensor for lane keeping assistance, a rear mounted ultrasonic sensor for blind spot detection, a forward mounted ultrasonic sensor for forward collision avoidance and speed adaption, as well as to provide the ability to perform intersection coordination. Furthermore, the 
Main Robot was connected to the computer to display the driver's LCD Panel. Robot A, Robot B, and Robot $\mathrm{C}$ were configured with a light sensor for Lane Keeping Assistance, a forward mounted ultrasonic sensor for Forward Collision Avoidance and Speed Adaption, and the ability to perform Intersection Coordination. The following programs were implemented to run the simulation:

- CADASClient.java - This program included the five core functions mentioned above. It was designed to control the Main Robot.

- CADASListener.java - This program was executed on the computer to interpret the robots sensed data and notify the driver.

- MyGUI.java - This program ran on the computer as well, and it acted as the driver's LCD Panel for display purposes for the Main Robot.

- RobA.java - This program contained the Lane Keeping Assistance, Forward Collision Avoidance and Speed Adaption, and Intersection Coordination functions for Robot A.

- RobB.java - This program was identical to RobA.java in functionality, with details pertinent to Robot B.

- RobC.java - This program was identical to RobA.java in functionality, with details pertinent to Robot C.

\subsection{Implemented functions}

\subsubsection{Lane keeping assistance}

Lane keeping assistance (LKA) was responsible for keeping the car in the lane by informing the driver every time they drifted while driving. In this simulation, the road was represented by black line. If the intensity value from the light sensor was greater than $40 \%$, then it meant the surface was understand as white. This implied that the robot had left the lane, and a notification was sent to the driver. However, if the intensity value was less than or equal to $40 \%$, then it meant the surface indicated black and implied the robot was within its lane. In the latter case, no notification was sent.

\subsubsection{Forward collision avoidance and speed adaption}

Forward collision avoidance and speed adaption (FCASA) function worked by checking ahead of the vehicle for obstacles and notifying the driver to slow down or stop depending on how close the object was. Objects were detected by using an ultrasonic sensor to determine whether or not an obstacle was within a legal or critical distance. If an obstacle was detected within the legal distance a notification and request to slow down was sent to the driver, similarly if the object was within the critical distance a notification was sent to the driver along with the request to stop. For the simulation purposes, the legal distance was set at $30 \mathrm{~cm}$, and the critical distance was $20 \mathrm{~cm}$.

\subsubsection{Blind spot detection}

Blind spot detection (BSD) worked by checking the blind spots around the vehicle for obstacles within the next lane by using an ultrasonic sensor. If obstacles were found, a notification was sent to the driver informing them that it was unsafe to change lanes. If obstacles were not found no action was provided by the system.

\subsubsection{Traffic sign recognition}

The primary task of Traffic Sign Recognition (TRS) was detecting and identifying traffic signs in order to notify the driver of the sign and suggest an action by using a color sensor. If the color sensor detected a red sign it interpreted that as a stop sign and a notification was sent to the driver with the 
suggestion to stop. Otherwise, if the color sensor detected a blue sign, it meant a slippery road sign had been encountered and the suggestion to reduce speed was sent along with a notification to the driver. A yellow sign indicated the vehicle had observed a school zone sign and a notification was sent to the driver with the recommendation to slow down. Lastly, if the color sensor detected a green sign, it meant an intersection sign had been detected and a notification was sent to the driver with the speed and arrival time of any other cars within the range of the intersection.

\subsubsection{Intersection coordination}

The purpose of Intersection Coordination (IC) was to facilitate safe and efficient intersection utilization among vehicles. As each robot approached the "intersection ahead" sign, a Bluetooth connection was established to a centralized computer attached to the intersection. Each robot submitted its speed to the central computer so that the time to reach the intersection could be calculated. For all the cars in range, the speed and time to reach the intersection was displayed to the driver.

\subsection{Challenging scenario}

The scenarios used a different number of robots and tested different functions. In this paper, we describe one scenario, which is most challenging one, because it involves four robots and five functions including the driver's LCD panel.

The demonstration for this scenario consisted of four robots, Main Robot, Robot A, Robot B, and Robot C, as shown in Fig. 7. Before the scenario started, all robots establish a Bluetooth connection with the CADASListener program. It should be noted that CADASListener also acts as a centralize computer for the Intersection Coordination. At the beginning of the scenario, Robot $\mathrm{C}$ reached the intersection alone, fully stopped, then continued driving along the line.

This scenario assumed that all the three robots, Main Robot, Robot A, and Robot B were in the range of an intersection travelling at different speed. One LCD Panel was used for the Main Robot, information was displayed in the following format: $<$ Rob, Speed, Time $>$. The parameters of the scenario allowed that the distance between an "intersection ahead" sign and the intersection itself remained constant. All three robots stopped when they reached the intersection. The strategy used was First-Come-First-Served (FCFS) to determine who should proceed through the intersection first.

The Main Robot reached the intersection first and continued on. Robot B reached the intersection second before continuing on. Lastly, Robot A reached the intersection last, and waited its turn before continuing. The strategy selected was FCFS. Another implemented case used random order because all the robots were set at the same speed, which made them, arrived to the intersection at the same time.

All robots used light sensors for Lane Keeping Assistance to ensure they stayed on the road. Similarly, all four robots were equipped with a forward mounted ultrasonic sensor for Forward Collision Avoidance and Speed Adaption function.

According to the Forward Collision Avoidance and Speed Adaption functionality, if an object was detected, the distance was measured. If that distance was less than the Legal distance, a notification alert of the object and a recommendation to slow down was sent to driver's LCD Panel. Secondly, if the distance was critical or less, a notification alert about the object with the suggestion to stop was sent to the driver's LCD Panel. Lastly, if the distance was greater than the legal distance, no action was provided. In either case, the robots reacted according to the recommendations.

Additionally, three traffic signs were dispersed randomly throughout the environment as shown in Fig. 7. Only the Main Robot was capable of detecting them by using a color sensor. For example, if the 


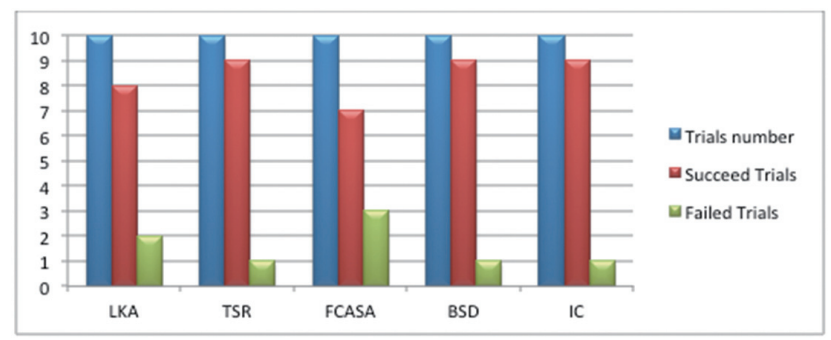

Fig. 8. The Scenario's result.

color sensor detected a red sign then the CADASClient notified the driver of a stop sign and reacted accordingly.

Lastly, there are two lanes in the environment in order to demonstrate Blind Spots Detection. The Main Robot was outfitted with a rear facing ultrasonic sensor and it travelled in the main lane. Robot C travelled in the secondary lane. Once they were beside each other, the Main Robot detected Robot A in the blind spot via the user interface to notify the driver, indicating it was unsafe to change lanes.

\subsection{Results}

This section provides a demonstration for five functions using four robots. These functions are Lane Keeping Assistance (LKA), Forward Collision Avoidance and Speed Adaption (FCASA), Traffic Signs Recognition (TSR), Blind Spots Detection (BSD), Intersection Coordination (IC). This scenario was in repeated trials.

Figure 8 shows the results for this scenario, which attempted to merge all functions and increase the number of robots. In general, most of the failed trials in this scenario happened due to lack of robot memory, which caused the robots to behave abnormally. FCASA function was failed three times in this scenario. The sensor, which was responsible for this function, was sending signals to the driver's LCD panel informing that there is an obstacle ahead while the road was clear. LKA function also failed twice because the light sensor suddenly stopped working due to unknown reasons [30].

Overall, the results illustrate that the system is able to help drivers to avoid accidents by providing suggestions and advices whenever they are necessary. This is the main goal of the proposed CADAS architecture. For example, when a driver has an intention to change lanes, the system checks the blind spot and decides whether it is safe or not. The addressed problems in this paper were tested except the system's processing time. This issue could not be tested due the limitation of the robots memory. Furthermore, the Main Robot was connected to the computer for display purposes while the rest of robots were connected to the same computer in the case of an intersection. These reasons prevented us from allowing the communication between vehicles for knowledge exchange purposes. However, the system demonstrates its ability to collect information for the area around using different types of sensors. In the IC function, the system is collecting data from traffic systems; it used recent information collected from all vehicles in the range of an intersection. The system plays alert messages; so senior drivers do not have to shift their focus from the road to the LCD panel. Finally, the simulation results showed that the simulation for the system architecture was successful based on the provided results, since no function failed less than $50 \%$. The system components, besides the sensors attached to the car, act as a decision maker to suggest the right actions to the driver and so help the driver avoid poor decisions. In order to avoid multiple actions appearing simultaneously, the installation of the system needs to be understood by the car's computer, for example, the blind spot detection should not work until the driver intends to use the car's change lane signals. 


\section{Conclusion and future work}

As more vehicles involved in the road, car accidents rates are increasing. An alert system has been developed to combat this issue. This paper presented a CADAS system architecture that consists of a great collection of functions to improve driver's behavior and decision making, thus will reduce car accidents. Since advanced driver assistance systems exist, CADAS system architecture takes into consideration traffic systems and senior drivers. It also attempts to use different types of sensors instead on relying on same types. The main components of CADAS are discussed and explained in detail. The interaction between drivers, vehicles, and the environment is the main concept behind the CADAS system, because it links drivers to their physical environment. Some functions of the proposed system were developed and demonstrated using a NXT robotic environment. In conclusion, the simulation showed that the proposed system architecture was successfully defined and has the ability to improve driver behavior and prevent them from making poor decisions. In future, we plan to use more enhanced robots and better quality sensors. We are also planning to use machine learning techniques to improve the proposed system decision making.

\section{References}

[1] M. Akhlaq, T. Sheltami, B. Helgeson and E. Shakshuki, Designing an integrated driver assistance system using image sensors, Journal of Intelligent Manufacturing (0956-5515) (2012), 1-24.

[2] M. Akhlaq and B. Helgeson, Development of an integrated driver assistance system using image sensors, 2010. Retrieved 11/5, 2010, from http://www.springerlink.com/content/1176578t3787v6k4/.

[3] M. Baldauf, S. Dustdar and F. Rosenberg, A survey on context-aware systems, International Journal of Ad Hoc and Ubiquitous Computing 2(4) (2007), 263-277.

[4] R. Bishop, Intelligent Vehicle Technologyand Trends, Artech House, Norwood, MA, 2005, pp. 25-37.

[5] Car Accidents Lawyers, Car accident death statistics, 2001. Retrieved 3/11, 2011, from http://www.car-accident-lawyersattorneys.com/car_accident_statistics.html.

[6] J.F. Coughlin, B. Reimer and B. Mehler, Driver wellness, safety \& the development of an AwareCar, Massachusetts Institute of Technology, 2009. Retrieved 2/11, 2011, from http://agelab.mit.edu/system/files/file/Driver_Wellness.pdf.

[7] D. Popovici, M. Desertot, S. Lecomte and T. Delot, When the context changes, so does my transportation application: VESPA, Procedia CS 5 (2011), 401-408.

[8] S. Fuchs, S. Rass, B. Lamprecht and K. Kyamakya, Context-awareness and collaborative driving for intelligent vehicles and smart roads, in: 1st International Workshop on ITS for an Ubiquitous ROADS. Piscataway, NJ, 6 July, 2007, pp. 1-6, CD.

[9] G. Zhao, K. Xuan, W. Rahayu, D. Taniar, M. Safar, M. Gavrilova and B. Srinivasan, Voronoi-based continuous k nearest neighbor search in mobile navigation, IEEE Transactions on Industrial Electronics 58(6) (2011), 2247-2257.

[10] O. Gietelink, J. Ploeg, B. De Schutter and M. Verhaegen, Development of advanced driver assistance systems with vehicle hardware-in-the-loop simulations, Vehicle System Dynamics 44(7) (2009), 569-590.

[11] H. Mousannif, I. Khalil and S. Olariu, Cooperation as a service in VANET: Implementation and simulation results, Mobile Information Systems 8(2) (2012), 153-172.

[12] E. Juliussen, Driver assistance system, the road ahead, Telematics Research Group Inc, 2008. Retrieved 5/17, 2011, from www.autofocusasia.com/electrical_elect-ronics/driver_assist_systems.htm.

[13] W. Katzman, Car accident lawyers in ontario: Windsor, sarnia and chatham offices, 2012. Retrieved 03/4, 2012, from http://www.katzman-wylupek.com/practice_areas/car-accident2.cfm.

[14] K. Xuan, G. Zhao, D. Taniar, M. Safar and B. Srinivasan, Voronoi-based multi-level range search in mobile navigation, Multimedia Tools Appl 53(2) (2011), 459-479.

[15] K. Xuan, G. Zhao, D. Taniar and B. Srinivasan, Continuous range search query processing in mobile navigation, in: Proceedings of the 14th International Conference on Parallel and Distributed Systems (ICPADS 2008), IEEE, 2008, pp. 361-368.

[16] Lane departure warning, 2012. Retrieved 02/28, 2012, from http://www.bmw.com/com/en/newvehicles/x/x6/2012/show room/comfort/lane_departure_warning.html.

[17] Lejos API, 2009. Retrieved 11/12, 2011, from http://lejos.sourceforge.net/nxt/nxj/api/index.html. 
[18] One World South Asia, Pedestrians and cyclists dying more in road accidents, 2009. Retrieved 20/3, 2011, from http:// southasia.oneworld.net/globalheadlines/pedestrians-and-cyclists-dying-more-in-road-accidents.

[19] Park assist, 2010. Retrieved 01/23, 2012, from http://www.bmw.co.uk/specials/5series_2010_showroom/com/en/newve hicles/5series/sedan/2010/showroom/comfort/parking_assistant.html\#media0.

[20] M. Pijpers, Sensors in ADAS. A literature study on the working principles and characteristics of frequently applied sensors in Advanced Driver Assistance Systems, Bachelor Thesis, University of Twente, 2007. Retrieved 12/28, 2010, from http://www.utwente.nl/ctw/aida/education/Rapport_MP.pdf.

[21] A. Rakotonirainy, Design of context-aware systems for vehicles using complex system paradigms, in: Workshop on safety and context in conjunction with CONTEXT'05, 158, 2005, pp. 43-51.

[22] A. Rakotonirainy and D. Steinhardt, In-vehicle technology functional requirements for older drivers. in: Proceedings of the 1st International Conference on Automotive User Interfaces and Interactive Vehicular Applications (AutomotiveUI '09), ACM, New York, NY, USA, 209, pp. 27-33, DOI=10.1145/1620509.1620515, from http://doi.acm.org/10.1145/ 1620509.1620515.

[23] M. Röckl, P. Robertson, K. Frank and T. Strang, An architecture for situation-aware driver assistance systems, in: Proceedings. IEEE 65th Vehicular Technology Conference - Spring 2007, April 22-25, 2007, Dublin, Ireland, ISBN 1-42440266-2, ISSN 1550-2252.

[24] S. Ilarri, E. Mena, A. Illarramendi, R. Yus, M. Laka and G. Marcos, A friendly location-aware system to facilitate the work of technical directors when broadcasting sport events, Mobile Information System 8(1) (2012), 17-43.

[25] J.P. Thalen, ADAS for the car of the future (Design Report), The Netherlands, Bachelor Thesis, University of Twente, 2005. Retrieved 01/07, 2011, from http://essay.utwente.nl/58373/.

[26] The LEGO Group, Mindstorms, 2011. Retrieved 09/09, 2011, from http://mindstorms.lego.com/en-us/Default.aspx.

[27] Thierry Delot, Nathalie Mitton, Sergio Ilarri, Thomas Hien, GeoVanet: A routing protocol for query processing in vehicular networks, Mobile Information Systems 7(4) (2011), 329-359.

[28] Thierry Delot, Sergio Ilarri, Nicolas Cenerario, Thomas Hien, Event sharing in vehicular networks using geographic vectors and maps, Mobile Information Systems 7(1) (2011), 21-44.

[29] Transport Canada (2012). Canadian Motor Vehicle Traffic Collision Statistics: 2009. Retrieved 09/06, 2012, from Government of Canada, http://www.tc.gc.ca/eng/roadsafety/tp-tp3322-2009-1173.htm.

[30] Yang Sun Lee, Jang Woo Park, Leonard Barolli, A localization algorithm based on AOA for ad-hoc sensor networks, Mobile Information System 8(1) (2012), 61-72.

Elhadi M. Shakshuki is a professor at the Jodrey School of Computer Science at Acadia University, Canada. He is the founder and the head of the Cooperative Intelligent Distributed Systems Group at the Computer Science Department, Acadia University. He received the BSc degree in Computer Engineering in 1984 from Tripoli University Libya, and the MSc and PhD degrees in Systems Design Engineering respectively in 1994 and 2000, from the University of Waterloo, Canada. Dr. Shakshuki is an Adjunct Professor at Dalhousie University, Canada. He is on the editorial board of several international journals and contributed in many international conferences and workshops with different roles. He published over 150 research papers in international journals, conferences and workshops. He is the founder of several conferences, symposia and workshops. He is a senior member of IEEE, and a member of ACM and APENS.

Wael Alghamdi holds the MSc from Jodrey School of Computer Science at Acadia University, Canada. He holds BSc of Computer Science from King Abduaziz University, Jeddah, Saudi Arabia in 2008. His current research focuses on Multi-agent Systems, Wireless Sensors Context-Aware system as well as Smart Homes Applications.

Tarek R. Sheltami is currently an associate professor at the Computer Engineering Department at King Fahd University of Petroleum and Minerals (KFUPM) Dhahran, Kingdom of Saudi Arabia. He joined the department on August 26, 2004. Before joining the KFUPM, Dr. Sheltami was a research associate professor at the School of Information Technology and Engineering (SITE), University of Ottawa, Ontario, Canada. He worked at GamaEng Inc. as a consultant on Wireless Networks (20022004). He was the Principle/Co Investigator of several research projects in the area of Ad hoc, Sensor Networks and Pervasive and Ubiquitous Computing. 

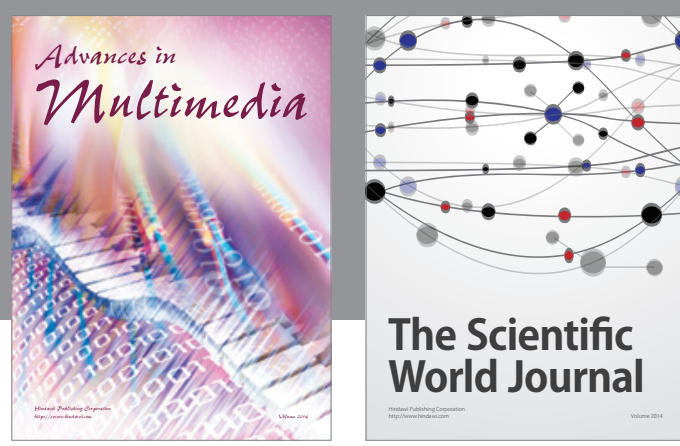

The Scientific World Journal
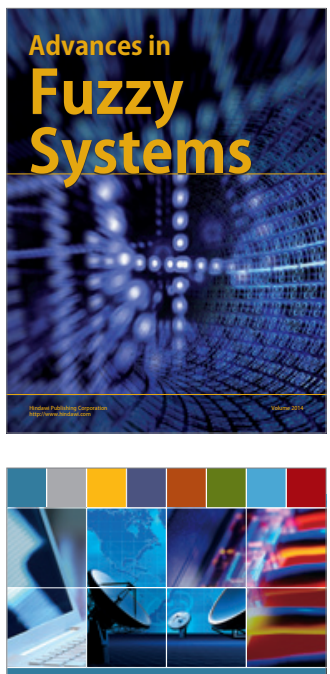

Computer Networks and Communications
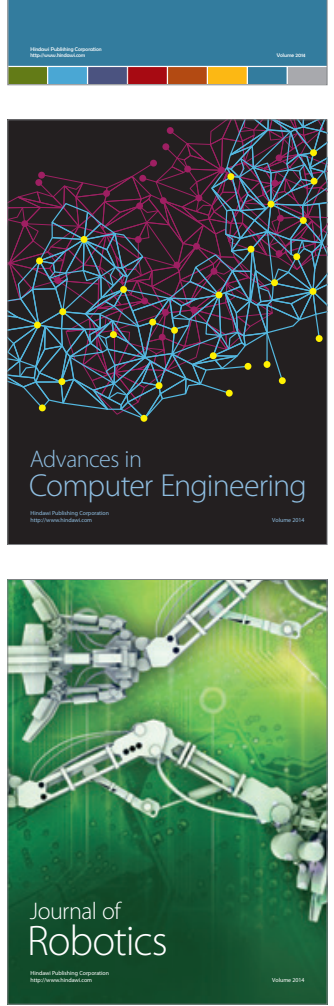
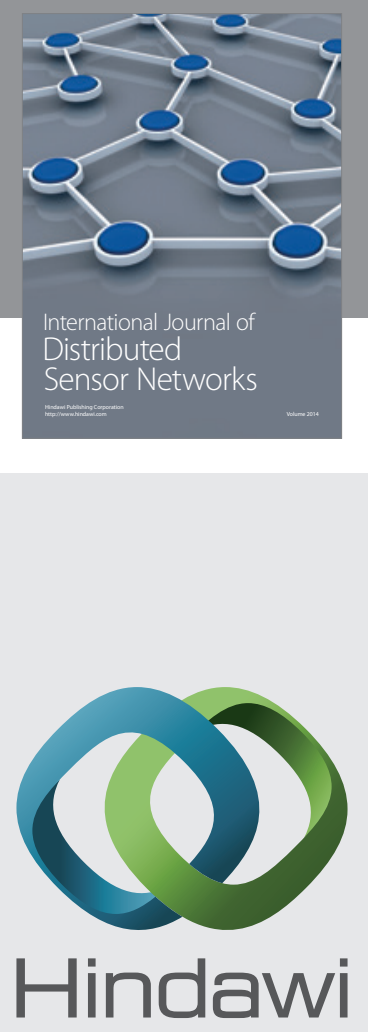

Submit your manuscripts at

http://www.hindawi.com
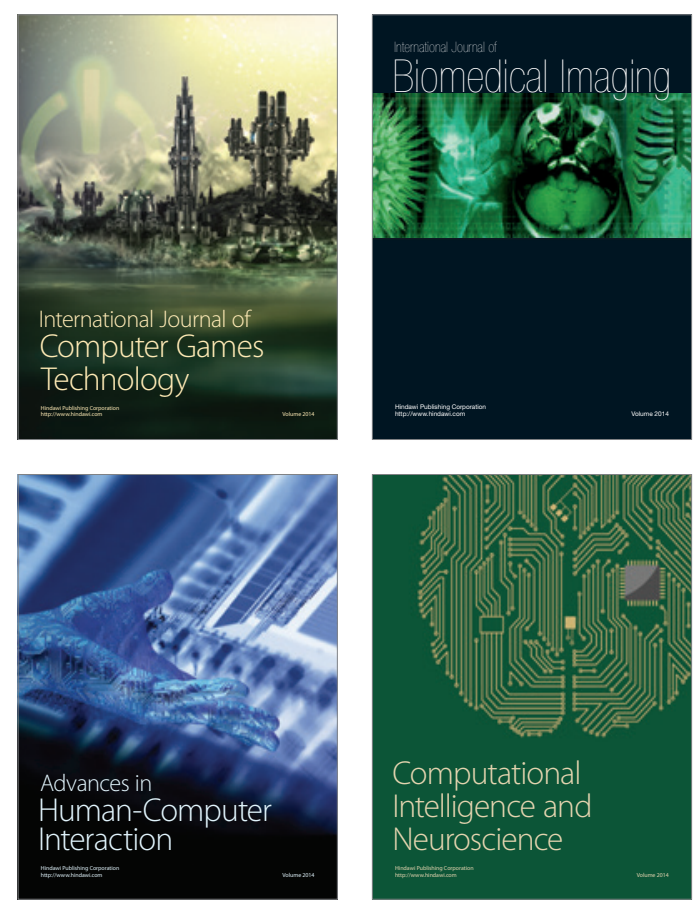
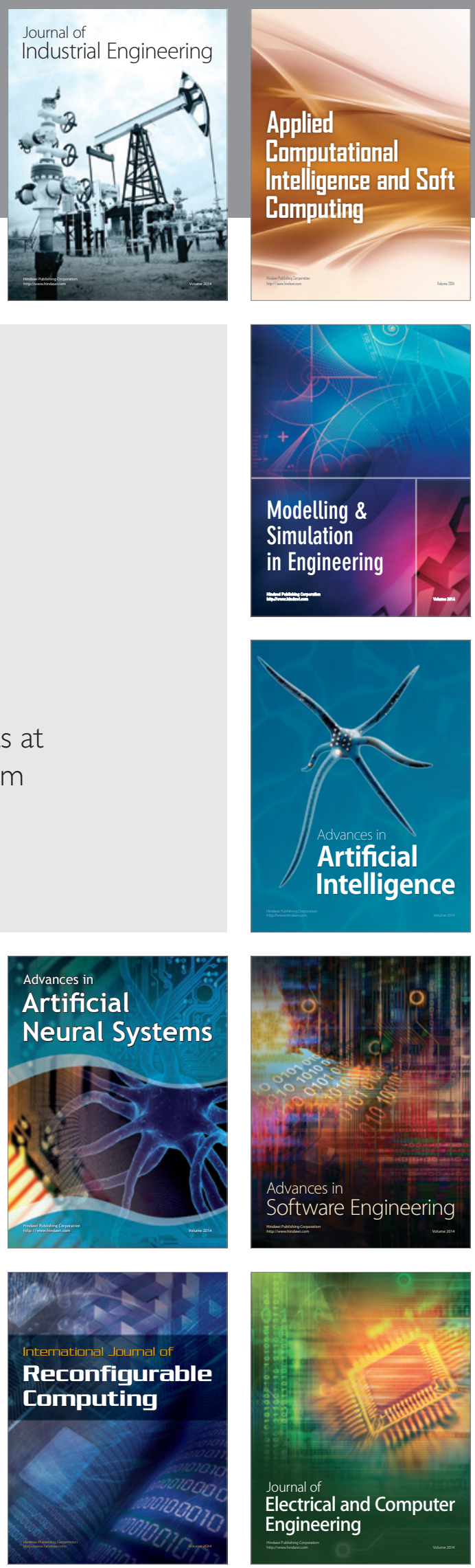